\title{
Protection contre \\ les affouillements de berge dans l'emprise des coudes des cours d'eau
}

\author{
PAR
}

M. Morin

SOGREAH, Grenoble
ET

M. Favant
Pont et Chaussées, ville nouvelle
d'Evry

\section{Introduction}

A la suite des inondations de l'Arc en 1957, où la vallée de la Maurienne fut endommagée par les divagations de cette rivière, la Direction Départementale de l'Equipement de Chambéry, avec l'aide financière du Service Central Hydrologique, décide de faire exécuter par SOGREAH, une étude générale des endiguements dans les coudes. Cette étude doit permettre de fixer, dans les projets, la cote de fondation des ouvrages de protection de berge, de telle sorte qu'ils ne soient pas déchaussés au moment des crues.

Elle est réalisée sur un modèle réduit à fond mobile de 1964 à 1970.

Le dépouillement des essais permet de dégager des courbes enveloppes de l'affouillement maximal correspondant à une série de coudes types.

Le but de la présente communication est l'application de ces résultats à un cas concret, ce qui a fait l'objet d'une note de synthèse remise à toutes les DDE et DDA de France par les soins du Service Central Hydrologique.

Dans un premier temps, nous allons rappeler rapidement les principales caractéristiques de l'étude qui ont fait l'objet d'une communication au Congrès de l'AlRH en 1971 à Paris (").

(*) XIVe Congrès de l'AlRH, 29 août all 3 septembre 1971. Volume 3 des comptes rendus; Sujet $C$ : «Déformation des lits alluvionnaires composés de matériaux à granulométrie étendue »; Sujet C, Comm. 16, E. Morin: "Dóformation des lits alluvionnaires dans les coudes des rivières $»$.

\section{1 - Rappel des caractéristiques et des résultats de l'étude}

\section{1 - MODËLE}

Le modèle est constitué par deux canaux de $1,5 \mathrm{~m}$ de large et de $40 \mathrm{~m}$ de long qui forment six coudes séparés par des sections droites de $6 \mathrm{~m}$ environ. L'angle de ces coudes est de $25^{\circ}, 45^{\circ}$ et $96^{\circ}$, tandis que le rayon de courbure du parement extérieur est de 4 et $10 \mathrm{~m}$.

\section{2. - MATÉRIAUX PENTES-DÉBIT}

Trois sables grossiers de granulométrie continue sont utilisés sur le modèle. Les pentes étudiées varient de 2,5 à $20 \%$; c'est dire que les rivières représentées sont du type rivière à galets.

Le modèle est équipé d'un circuit fermé à matériaux et les mesures sont effectuées pendant le passage de l'eau lorsque l'état d'équilibre est atteint dans chacun des coudes.

Pour une pente et un matériau donnés, toute une gamme de débits est essayée.

\section{3 - PROTECTION}

Trois types de protections sont mises en place sur le modèle :

- les digues verticales pour lesquelles une série d'essais très complète est exécutée;

- les perrés inclinés;

- les digues verticales avec radier de pied. 

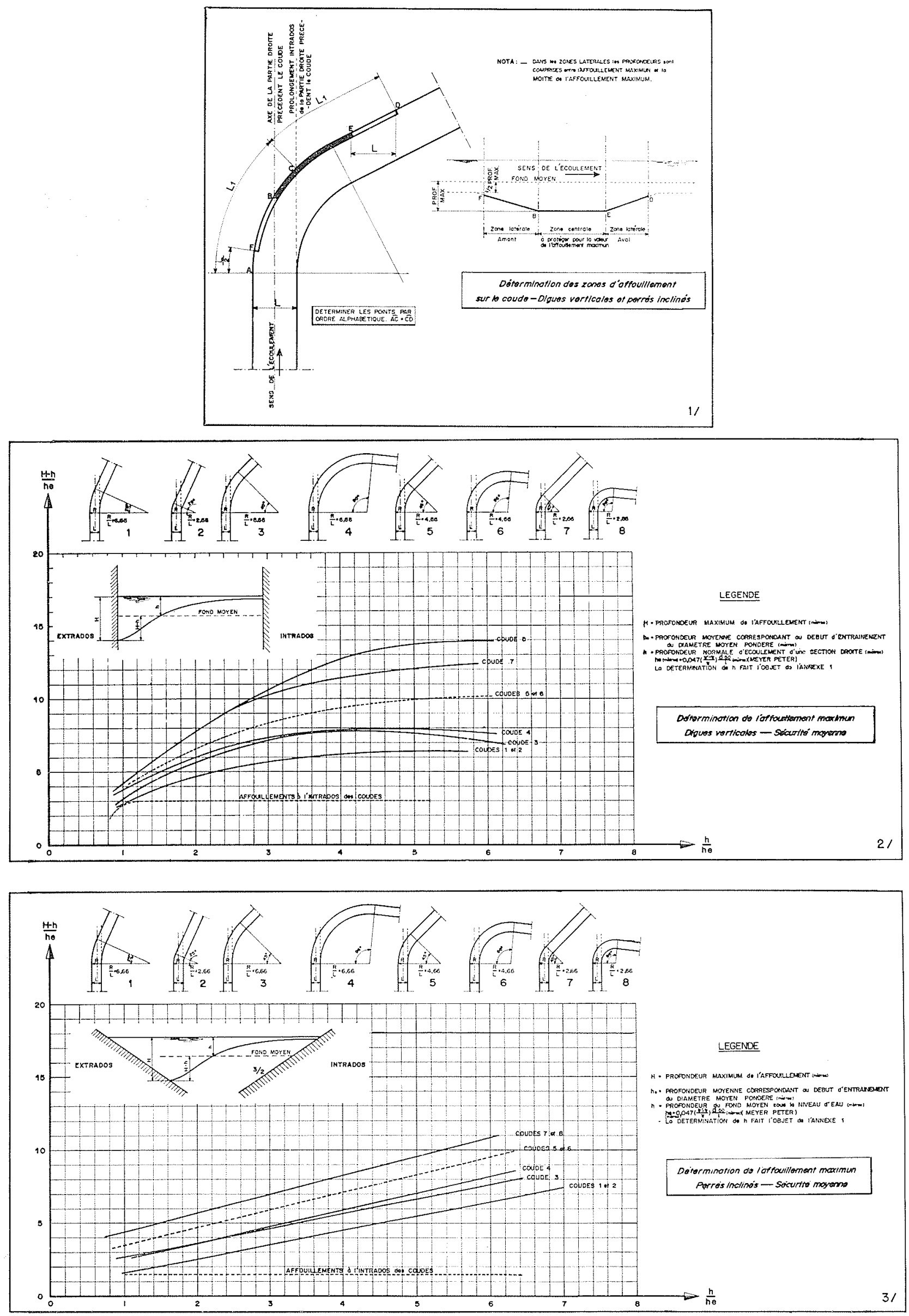


\section{4 - MESURES EFFECTUÉES}

Les principales mesures effectuées sont la prise de profils en travers du canal, fond et ligne d'eau environ tous les mètres linéaires de canal.

Ces profils en travers sont relevés pendant le passage de l'eau grâce à deux appareils, l'un qui palpe le fond, l'autre la surface de l'eau.

Par ailleurs, l'évolution de l'affouillement dans le coude est aussi étudiée par la mise en place d'un tâte-fond en un point fixe, tâte-fond qui enregistre le passage des bancs du transport solide.

\section{5 - DÉPouILLEMENT}

L'ensemble des essais représente un nombre important de mesures; l'ordinateur a donc été utilisé pour leur dépouillement.

Il s'avère difficile toutefois de trouver des formules qui puissent donner l'affouillement en fonction des caractéristiques du coude. En effet, la largeur du canal, le rayon de courbure du coude et son angle interviennent simultanément de façon compliquée.

De ce fait, nous nous sommes orientés vers une formulation simple par coude qui permettra une exploitation facile.

\section{2 - Exploitation des résultats}

\section{1 - DOMAINE DE VALIDITÉ}

Si l'on tient compte des conditions d'expérimentation, ces résultats ne sont applicables qu'aux rivières à galets dont la pente varie entre $1 \%$ et $25 \%$. Il serait intéressant toutefois de regarder s'ils ne sont pas aussi applicables à des rivières de pente très faible.

L'affouillement indiqué par les courbes est celui résultant du méandrement naturel de la rivière et de l'effet de coude. On suppose que le fond moyen du lit reste à un niveau constant.

Aux profondeurs trouvées, il faudra éventuellement ajouter la valeur de l'abaissement général du lit ou retrancher son relèvement. Ces mouvements généraux du lit ont d'autres causes, comme les emprunts des graviers, la mise en place d'un seuil sur le lit ou la mise en place de l'endiguement lui-même.

\section{2 - PRÉSENTATION DES RÉSULTATS}

\subsection{1 - Position de l'affou:llement sur le coude (Graphique 1)}

L'expression mathématique de la position de l'affouillement sur le coude est compliquée, aussi lui avons-nous préféré le petit schéma ci-après qui s'adapte à tous les coudes de rivière, et est valable pour la plupart des protections, à l'exception des digues verticales avec radier de pied où la zone d'affouillement maximale se prolonge plus loin vers l'aval.

\subsection{2 - Affouillement maximum (Graphiques 2 et 3)}

La valeur de l'affouillement maximum est donnée par des courbes-enveloppes, chacune se rapportant à un coude caractéristique.

Une similitude, amenant la largeur de la rivière à celle des coudes indiqués sur la graphique, permet de connaître rapidement quel est le coude qui correspond au cas concret que l'on étudie.

On se reporte suivant le type de protection à la courbe caractéristique du coude.

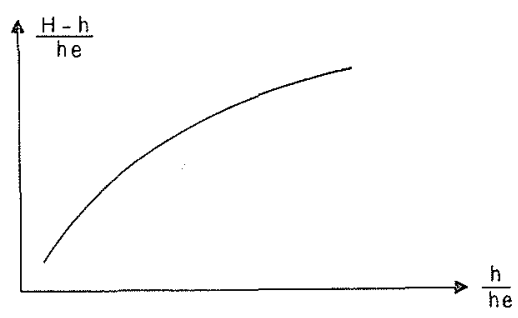

En abscisse on trouve le rapport h/he où :

$$
h e==0,047\left(\frac{\Upsilon^{\prime}-\underline{Y}}{\gamma}\right) \frac{d_{50}}{i}
$$

he représente la profondeur moyenne, correspondant au début d'entraînement;

$\gamma^{\prime}$ et $\gamma$ sont respectivement les poids spécifiques du matériau et de l'eau;

$d_{50} \quad$ est le diamètre moyen pondéré:

$i$ est la pente de la ligne d'énergie généralement égale à la pente de la rivière endiguée.

Ce terme indique en fait pour le débit considéré l'état de la rivière par rapport au début d'entraînement des matériaux du fond.

En ordonnée, on trouve:

$$
(H-h) / h e
$$

où $H-h$ est la profondeur maximale de l'affouillement sous le fond moyen.

Les graphiques indiquent donc les profondeurs maximales des affouillements à prendre en compte pour le coude et Ies débits envisagés.

\section{3 - DÉTERMINATION DE 《h»}

Une fois connues la granulométrie du matériau et la pente de la rivière, on détermine facilement la valeur de he.

La valeur de $h$ peut être plus difficile à obtenir.

On part de la définition suivante:

Il est entendu que l'on considère la rivière endiguée à l'amont avec la même largeur que dans le coude. $h$ représente alors la profondeur moyenne dans une section amont à l'approche du coude. $h$ est calculé en supposant que tout l'écoulement se répartit uniformément sur toute la largeur entre digues.

$$
h=S / L
$$

si :

$S$ est la section mouillée réelle de l'écoulement;

$L$ est la largeur entre digues. 
I eas

On connaît la section mouillée pour le niveau de crue que l'on veut considérer. La détermination de $h$ est immédiate.

\section{$2^{\circ}$ cas}

On connaît la loi hauteur-débit jusqu'à un débit inférieur à celui que l'on veut considérer. On extrapole cette loi jusqu'au débit recherché à partir des sections mouillées, des rayons hydrauliques et de la pente par la formule de Strickler :

$$
Q=K S R^{213} J^{1 / 2}
$$

où :

$K$ est donné par les valeurs connues de la loi hauteur-débit.

\section{$3^{e}$ cas}

On ne possède aucune observation de niveaux ou de débits.

A partir de profils en travers on établira les relations section et rayon hydraulique en fonction du niveau.

On utilisera ensuite la formule de Strickler précitée:

$$
Q=K S R^{2 / 3} J^{1 / 2}
$$

Le plus difficile est alors de déterminer le coefficient de rugosité qui est fonction d'un grand nombre de facteurs, mis ici par ordre d'importance pour les rivières endiguées:

- la rugosité de peau, c'est-à-dire celle produite par les matériaux à la surface du lit;

- la rugosité de forme du fond (lits mineurs, bancs, dunes);

- l'alignement et le parallélisme des berges;

- la rugosité propre aux berges dont l'effet est d'autant plus important que le rapport de la largeur du lit à la profondeur est faible.

On exprime le coefficient global $K$ en fonction du coefficient de rugosité de peau $K^{\prime}$ et des autres caractéristiques du lit exprimées chacune par un coefficient $h$.

On aura:

$$
K=K^{\prime}\left(1-n_{1}-n_{2}-n_{3}\right)
$$

$K^{\prime}$ est donné par:

$$
K^{\prime}=\frac{21}{\left(d_{\text {50 }}\right)^{1 / 6}} \text { ou } \frac{26}{\left(d_{90}\right)^{1 / 6}}
$$

où $d_{50}$ et $d_{90}$ sont, en mètres, les diamètres moyens pondérés.

Les valeurs de $n_{1}, n_{2}$ et $n_{3}$ sont données par le tableau ci-contre.

Si les résultats sont très différents avec l'un et l'autre diamètre, on calculera l'affouillement dans les deux cas,

\begin{tabular}{|c|c|c|c|}
\hline \multirow{5}{*}{$n_{1}$} & 0 & 0,1 & Lit très plat et très régulier \\
\hline & 0,1 & 0,2 & $\begin{array}{l}\text { Lit mineur formant de longues sinu- } \\
\text { soïdes entre bancs longs à surface } \\
\text { régulière }\end{array}$ \\
\hline & 0,2 & 0,3 & $\begin{array}{l}\text { Lit mineur divisé en plusieurs bras } \\
\text { entre bancs à surface relative- } \\
\text { ment régulière }\end{array}$ \\
\hline & 0,3 & 0,4 & $\begin{array}{l}\text { Lit mineur divisé entre bancs à } \\
\text { surface irrégulière (bancs en } \\
\text { écaille, dunes, ripples...) }\end{array}$ \\
\hline & 0,4 & 0,5 & Lit très tourmenté \\
\hline \multirow{3}{*}{$n_{2}$} & 0 & 0 & $\begin{array}{l}\text { Berges très rectilignes et très pa- } \\
\text { rallèles }\end{array}$ \\
\hline & 0,05 & 0,1 & $\begin{array}{l}\text { Tracé général parallèle mais légè- } \\
\text { rement sinusö̈dal à longueur } \\
\text { d'onde assez grande }\end{array}$ \\
\hline & 0,15 & 0,25 & $\begin{array}{l}\text { Tracé très sinueux ou largeur rapi- } \\
\text { dement variable et irrégulière sur } \\
\text { une assez grande longueur }\end{array}$ \\
\hline \multirow{4}{*}{$n_{3}$} & $-0,1$ & $-0,2$ & $\begin{array}{l}\text { Berge très lisse, lit étroit vis-à-vis } \\
\text { de la profondeur d'eau }\end{array}$ \\
\hline & 0 & 0 & $\begin{array}{l}\text { Berge lisse, lit large, ou berge régu- } \\
\text { lière avec même rugosité de } \\
\text { «peau } » \text { que le fond }\end{array}$ \\
\hline & 0,05 & 0,1 & $\begin{array}{l}\text { Berge rugueuse par rapport au fond, } \\
\text { lit large }\end{array}$ \\
\hline & 0,1 & 0,2 & $\begin{array}{l}\text { Berge rugueuse par rapport au fond, } \\
\text { lit étroit }\end{array}$ \\
\hline
\end{tabular}
car cet écart sera atténué déjà sur $h$.
Valeur de $n_{1}, n_{2}, n_{3}$

dams la formule $K=K^{\prime}\left(1-n_{1}-n_{2}-n_{3}\right)$

\section{3 - Résultats généraux}

I a comparaison des résultats entre perrés inclinés et digues verticales reste très en faveur des perrés inclinés, résultat qui est bien connu. Les résultats permettent une comparaison économique intéressante.

Des essais sont aussi effectués avec des perrés inclinés, rugueux. Ils conduisent aux mêmes profondeurs d'affouillement que les perrés lisses.

L'étude permet d'estimer l'affouillement possible à l'intérieur d'un coude, ou même sur une partie droite uniquement exposée au méandrement naturel.

Cet affouillement varie entre 1,5 et 4 fois he sous le fond moyen suivant le type de protection envisagé et le degré de sécurité exigé. 


\section{Discussion}

Prósident : M. J. CHAPON

M. le Président remercie les auteurs de cette communication précise et dense qui, dit-il, présente deux caractéristiques: d'une part, elle analyse le phénomène naturel et, d'autre part, elle va jusqu'à l'application, à des applications très précieuses, ainsi que le montre le dernier exemple que M. Morin vient de nous exposer.

C'est une étude qui a débouché sur des résultats concrets, et je profite de l'occasion pour remercier ses auteurs au nom de l'Administration à laquelle j'appartiens et aussi au nom de celle de l'Agriculture, car cette recherche a permis de donner aux Services un outil pratique pour travailler.

C'est précisément sur cet aspect des choses que je voudrais ouvrir la discussion. Quelqu'un a-t-il des observations à présenter, en particulier sur la facilité d'utilisation de cette méthode, sur le choix de la valeur numérique des paramètres qu'elle utilise, etc.

La diffusion de la méthode est encore à son début, répond M. Verdet, mais, à la SOGREAH, nous l'utilisons fréquemment - en liaison avec M. Morin - pour diverses études.

M. LARINIER (Laboratoire du CTGREF, Besançon) pose au conférencier la question suivante:

Vous utilisez le critère de Shields de début d'entraînement, basé sur l'utilisation du $d_{50}$, déterminé sur la courbe granulométrique du matériau de fond. Ne pensez-vous pas que, pour les rivières où l'on observe un phénomène de «pavage», il serait préférable d'utiliser le $d_{30}$, comme le propose Kellershals?

Le $d_{n 0}$ est effectivement le critère du début d'entraînement, réponc M. Morin. Mais, dans le cas qui nous occupe où il y a entraínement global d'une couche plus ou moins épaisse du matériau de fond, il est apparu que le $d_{50}$ est un critère préférable; le terme he/h n'est qu'un paramètre de comparaison entre le tirant d'eau existant dans la rivière et celui correspondant au début d'entraînement. En effet, pour les rivières à fond mobile de galets - auxquelle se limite notre étude - lors des crues décennales, au voisinage de l'endiguement des berges, les matériaux d'une granulométrie de l'ordre de $d_{80}$ sont rapidement balayés et on assiste à un entraînement «global» des matériaux de fond qui ne se limite pas à la couche superficielle de ces derniers.

Sur une nouvelle question de M. Larinier, M. Morin indique que, lors des essais sur le modèle du coude, on a souvent observé des dunes et des rides sur le fond; cette question a été spécialement étudiée par M. Djedidi qui en a fait l'objet d'une thèse et a défini les divers critères d'apparition des dunes au moyen d'équations mathématiques assez compliquées. Par mesure de simplification, le Service Central Hydrologique a estimé qu'il convenait de ne pas faire état de ces critères dans la note de synthèse destinée aux applications pratiques.

Au fur et à mesure que l'affouillement se poursuit, observo M. le Président, les caractéristiques granulométriques du fond se modifient; ne faut-il pas en tenir compte?

Il ne le semble pas, répond $M$. Morin. Comme dans d'autres expériences sur les affouillements (autour des piles de pont, pa exemple), nous avons observé que le maximum de l'affouillement se produit pour des débits peu supérieurs au débit de commencement d'entraînement. En première approximation, on peut expliquer ce fait par le remblaiement partiel de l'affouillement par l'accroissement du débit solide provenant de l'amont, lorsque le débit liquide augmente.

Comment utiliser vos abaques, interroge M. le Président, lorsque les berges d'un canal sont inclinées, mais prolongées à leur partic inférieure par un rideau vertical de palplanches?

Il paraît prudent de ramener ce cas à celui de berges verticales, répond $\mathrm{M}$. MoRIN.

M. le Président remercie M. Morin et tous ceux qui ont animé la discussion et donne la parole à M. Cocozza pour l'exposé de sa communication. 